Die Voraussetzung der beschleunigten Umgebung bei ruhendem Karussell scheint demnach mit der Tatsache, daß man auf dem bewegten Karussell bei Tage die Sonne und Abends die Sterne sämtlich in demselben Augenblick eine beschleunigte Bewegung annehmen sieht, wie die nächste Umgebung auf der Erde, unvereinbar.

4. Damit aber noch nicht genug. Wenn man nicht die ganz absurde Annahme machen will, daß der Sternhimmel sich »zufällig « jedesmal genau in demselben Augenblick in Bewegung setzt und wieder zur Ruhe kommt, wie das Karussell, so muß man einen ursächlichen Zusammenhang annehmen zwischen der Kraft, welche auf das Karussell einwirkt, und der Bewegung. der Himmelskörper. Nun breitet sich die Kraft nach Einstein ebenfalls mit Lichtgeschwindigkeit aus, sie braucht also bis zur Sonne 8 Minuten, bis zu den Fixsternen Jahre, Jahrhunderte. Die oben unter 3. genannten Verzögerungen müssen also alle verdoppelt in die Erscheinung treten, wenn man das aligemeine Relativitätsprinzip auf die be- schleunigte Drehbewegung anwenden will. Daß unter den gemachten Annahmen die Sonne eine Geschwindigkeit haben würde, die das 200-fache des Lichtes betrüge, und daß sie folglich in der Bewegungsrichtung ebenfalls zu einer Linie zusammenschrumpfen müßte, sei hier nur nochmals erwähnt.

Was diese Einwände so außerordentlich schwer erscheinen läßt, ist der Umstand, daß sie auf ganz alltäglichen, leicht beobachtbaren Tatsachen beruhen, in Verbindung mit den ebenfalls exakt meßbaren Erscheinungen der zeitlichen Ausbreitung des Lichtes und der Entfernung der Himmelskörper. Die unter $\mathbf{r}$. benutzte Verkürzung in der Bewegungsrichtung und die unter 4. hinzugezogene zeitliche Ausbreitung der Gravitation sind wesentliche Bestandteile der Einsteinschen Theorie.

Es scheint demnach, daß sehr offen daliegende Tatsachen der allgemeinen Relativitätstheorie widersprechen:

Valkenburg, I92 I Januar.

\title{
Photometrische Sternfolge in der Umgebung der Nova Cygni.
}

Während der Verfolgung der Lichtabnahme der Nova im Oktober und November 1920 sind in der Nachbarschaft die folgenden Sterne an einem Vergleichskeilphotometer beobachtet worden.

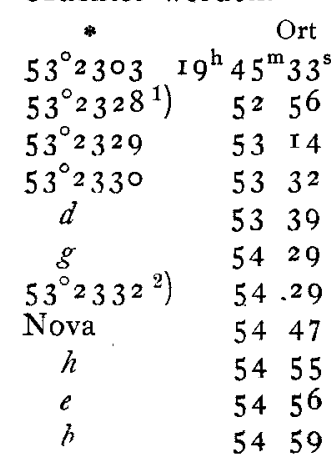

.

\begin{tabular}{|c|c|c|}
\hline \multicolumn{2}{|c|}{ oacht } & $\mathrm{Gr}$. \\
\hline \multirow{2}{*}{\multicolumn{2}{|c|}{$\begin{array}{c}9.73,73,59,87 \\
6 \text { Beob. }\end{array}$}} & $9^{m} \cdot 73$ \\
\hline & & 10.73 \\
\hline \multicolumn{2}{|c|}{ Io Beob. } & 8.47 \\
\hline \multirow{2}{*}{\multicolumn{2}{|c|}{$\begin{array}{c}9.68,82,87,77 \\
6 \text { Beob. }\end{array}$}} & 9.78 \\
\hline & & 10.07 \\
\hline \multirow{2}{*}{\multicolumn{2}{|c|}{$\begin{array}{l}8.09,97,10,15, \circ 5 \\
\text { (Harv. Syst.). }\end{array}$}} & 8.07 \\
\hline & & \\
\hline $\begin{array}{l}\text { Harv. } \\
7^{\mathrm{m}} \times 6\end{array}$ & $\begin{array}{l}\text { Potsd. red. } \\
7^{m} \cdot 47\end{array}$ & \\
\hline & 7.67 & \\
\hline 8.02 & 7.93 & \\
\hline 7.77 & $8.0 \mathrm{I}$ & \\
\hline 7.02 & 8.16 & \\
\hline
\end{tabular}

Bergedorf, r920 Nov. 20.

1) tief gelb. $\left.{ }^{2}\right)$ Gesamtlicht mit dem engen Nachbar $53^{\circ} 233 x^{a}$.

\section{Zwei bewegte Sterne beim Triangulumnebel.}

Bei Vergleichung mehrerer Reflektor-Aufnahmen des Nebels M 33 fand ich zwei bewegte Sterne, die zwar keine große Bewegung besitzen, die aber wegen ihrer Lage bei einem der größten Spiralnebel und wegen der Gleichheit ihrer Bewegung Interesse verdienen. Ich maß die Örter der Sterne, auf einer Platte vom Bruce-Refraktor:

$$
\begin{aligned}
& \text { Nr. } \alpha_{1875} \quad \delta 1875 \\
& \text { I06 } 3 \mathrm{I}^{\mathrm{h}} 25^{\mathrm{m} 8 \mathrm{~s}} 48+30^{\circ} \cdot 6^{\prime} 27^{\prime \prime} 6 \text { gegen } * * \mathrm{I} \text { und } 2 \\
& \text { I } 064 \text { I } 267.32+293224.8 \text { gegen } * * \text { I tnd } 3
\end{aligned}
$$
$*$ I $=$ CbrE. 85 ז, *2 $=$ Lei $564, * 3=$ CbrE. 869. Epoche I 902.73 . Der erste Stern ist etwa $\mathrm{I}^{\mathrm{m}} \cdot 7$, der $z$ weite etwa $\mathrm{I} \circ \mathrm{m} \cdot \mathrm{O}$.
Die Bewegung ist auf den Reflektor-Aufnahmen nur mit ziemlicher Unsicherheit zu messen, weil die extraaxialen Bilder bei ihrer Verzerrung. solches nur roh zulassen. Ich finde für beide Sterne die Bewegung:

$$
A s=0 \text {." II2 } \varphi=85^{\circ} \text { Epoche т 9 4.I. }
$$

Über die Umgebung der Sterne wäre noch zu bemerken :

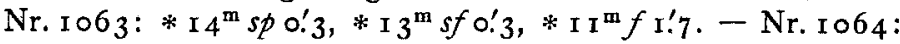
* I $3^{\mathrm{m}} n$ o. $4, * \mathrm{I}_{2}{ }^{\mathrm{m}} s$ o. 9 ; ein Nebelknoten mit angehängtem Sternchen geht nördlich I!́ I voran.

Königstuhl, I920 Aug. 20.

\section{$Z$ wei bewegte Sterne in Pisces.}

Kürzlich stießen mir auf Sechszöllerplatten die beiden kräftiger bewegten Sterne auf:

Nr. I066 $9^{\mathrm{m}} \cdot 3 \quad \alpha_{1875}=\mathrm{I}^{\mathrm{h}} 3^{\mathrm{I}^{\mathrm{m}} 25^{\mathrm{s}} \cdot 4} \quad \delta_{1875}=+17^{\circ}$ I $^{\prime} \cdot 3$

$$
1065 \text { I-1.0 I } 3610.76 \text { t } 163034^{\prime \prime} .9 \text {. }
$$

Den Ort des ersten, welcher $=\mathrm{BD}+\mathrm{I}^{\circ} 235$ ist, entnahm ich der B. D.; den zweiten vermaß ich auf einer Sechszöllerplatte der Epoche 1920.70 durch Anschluß an Berl A-487
1. 493. Die Bewegungen, mehr geschätzt als gemessen, sind:

$$
\begin{array}{rr}
\text { Nr. } 1066 \\
\text { 1065 }
\end{array} \quad A s=\begin{aligned}
& 0.42 \\
& 0.48
\end{aligned} \quad \varphi={ }_{\text {I }} 40^{\circ} \quad \text { Epoche 1912.2 }
$$

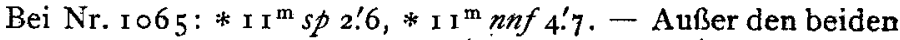
fand ich auch wieder den bewegtèn Stern Nr.8 I (AN 202.162), dessen Bewegung damit bęstätigt wird.

Königstuhl, I920 Sept. I 4 .

M. Wolf. 